\title{
Rigid Point-Surface Registration Using an EM Variant of ICP for Computer Guided Oral Implantology
}

\author{
Sébastien Granger ${ }^{1,2}$, Xavier Pennec ${ }^{1}$, and Alexis Roche ${ }^{1}$ \\ 1 INRIA, Epidaure Project, Sophia Antipolis, France \\ 2 AREALL, Neuilly-sur-Seine, France \\ \{Sebastien.Granger, Xavier.Pennec, Alexis.Roche\}@sophia.inria.fr
}

\begin{abstract}
We investigate the rigid registration of a set of points onto a surface for computer-guided oral implants surgery. We first formulate the Iterative Closest Point (ICP) algorithm as a Maximum Likelihood (ML) estimation of the transformation and the matches. Then, considering matches as a hidden random variable, we show that the ML estimation of the transformation alone leads to a criterion efficiently solved using an Expectation-Maximisation (EM) algorithm. The experimental section provides evidences that this new algorithm is more robust and accurate than ICP and reaches a global accuracy of $0.2 \mathrm{~mm}$ with computation times compatible with a peroperative system.
\end{abstract}

\section{Introduction}

Oral implantology is a domain where computer guided surgery can lead to drastic improvements in safety and quality of the operation for the patient. The operation is planned on a preoperative CT-Scan and the purpose of such a system is to help the dentist to drill the implant in the predefined position and orientation. The DentalNavigator system (patent pending), developed by AREALL [5], is a peroperative system based on surface registration. In the CT-Scan image, the teeth and jaw bone surfaces are segmented using a Marching-Cube algorithm resulting in about 100000 triangulated points. Points on the same structures are measured on the patient using an ultrasound sensor mounted on a passive robotic arm. This time we get between 50 and 1000 unstructured points. After the registration, the US sensor is replaced by the drill on the robot arm and the system visually guides the surgeon to the planned position and orientation for drilling. In this article, which is a short version of research report [15], we investigate the registration step of this system.

The registration of two sets of points is usually performed using one of the multiple variations around the ICP algorithm [9,14]. Many variants and improvements of this algorithm have been proposed: features more complex than points, Mahalanobis distance to take heteroscedastic (non isotropic, non-homogeneous) noise into account, use of robust estimators for outliers rejection, etc [14,13,11]. In almost all these variants, each scene point is matched with only one model 
point with an implicit constant weight. Moreover, sudden changes in the closestpoint function lead to a highly non-convex energy function, full of local minima. An improvement consists in using multiple weighted matches for each scene point: Rangarajan et al. introduced a probabilistic vision of the matching problem, and developed smooth point-matching models based on Gaussian weight (SoftAssign [1]) and Mutual Information [2], leading to a smaller number of local minima and thus presenting a better accuracy and robustness.

Starting from ICP, our main motivation was to improve the accuracy and the robustness in scope of a real-time system. We experimentally observed that Rangarajan's algorithms [1,2] were only efficient for registering two comparable sets of points (e.g. landmarks or surface equally sampled). Our problem is slightly different because the peroperative set of points is highly sub-sampled compared to the segmented surface and scene points are sparse enough to be considered as independent. Following Rangarajan's probabilistic approach, we develop in section [2] a registration criterion based on EM principles. The same criterion, inspired from [12, was independently developed in [7, but the derivation and use of our algorithm is original. Moreover, in section 3, we demonstrate new important properties leading to an efficient implementation of the algorithm. Finally, we discuss in section 4 the experimental results on our oral implantology application in terms of robustness, internal and global accuracy.

\section{Maximum Likelihood Estimations of the Transformation}

In this section, we model the scene as a random process, and we show that a maximum likelihood estimation of the transformation and the matches leads to the ICP algorithm using the Mahalanobis distance. Then, we consider the matches as a random matrix (or the model as a mixture of Gaussians) and we search for the ML estimate knowing only the transformation. Finally, we show how to solve efficiently this last criterion using an EM algorithm. This framework can be easily robustified by adding a probability to match a scene feature to the background [12, pp.78], which amounts to thresholding the Mahalanobis distance.

\subsection{Maximum Likelihood and Standard ICP}

Let $s_{i}$ be the features of the scene $\mathcal{S}, m_{j}$ the features of the model $\mathcal{M}, \mu^{2}$ the Mahalanobis distance between features and $T$ a rigid transformation from the model to the scene. Assuming that $s_{i}$ is homologous to $m_{j}$ (a measure of $T \star m_{j}$ ) with an additive Gaussian noise, its density probability function is:

$$
p\left(s_{i} \mid m_{j}, T\right)=k^{-1} \cdot \exp \left(-\mu^{2}\left(s_{i}, T \star m_{j}\right) / 2\right)
$$

Because we deal later on with multiple and weighted matches, we use a matrix $A$ to represent matches estimation where $A_{i j}=1$ if $s_{i}$ matches $m_{j}$ and 
0 otherwise. Since each scene point $s_{i}$ is assumed to correspond exactly to one model point with index say $j^{\star}$, we have $A_{i j}=\delta_{j j^{\star}}$ and $\sum_{j} A_{i j}=1$ for all scene index $i$. As $\alpha^{1}=\alpha$ and $\alpha^{0}=1$, we can write the conditional pdf of $s_{i}$ as: $p\left(s_{i} \mid A, \mathcal{M}, T\right)=\prod_{j}\left(p\left(s_{i} \mid m_{j}, T\right)\right)^{A_{i j}}=p\left(s_{i} \mid m_{j^{\star}}, T\right)$. Now, assuming that all scene points are conditionally independent, the scene likelihood is:

$$
p(\mathcal{S} \mid A, \mathcal{M}, T)=\prod_{i} p\left(s_{i} \mid A, \mathcal{M}, T\right)=\prod_{i j}\left(p\left(s_{i} \mid m_{j}, T\right)\right)^{A_{i j}}
$$

Taking the negative log, we obtain the following criterion to be maximised:

$$
C(T, A)=\frac{1}{2} \sum_{i j} A_{i j} \cdot \mu^{2}\left(s_{i}, T \star m_{j}\right)+N_{\mathcal{S}} \cdot \log k
$$

One recognises here the standard ICP criterion using the Mahalanobis distance. This proves that ICP maximises the scene likelihood under a Gaussian noise with exact correspondences. Moreover, [8] showed that this is the best (minimal variance) estimator. Here, the criterion is invariant w.r.t a global scaling of the noise variance. This property will not hold for the following EM formulation.

\subsection{Maximum Likelihood with Uncertain Matches}

In the previous section, the transformation and the matches were both estimated by directly maximising the scene likelihood knowing these variables. In fact, we only need to determine the transformation for our application, and the matching matrix is an auxiliary variable. Moreover, there can be ambiguities in the matching estimation and considering multiple matches amounts to seeing the scene points as measurements of a mixture of Gaussians around the model points. This interpretation is specially adapted for our case since the model is a surface and not a collection of landmarks. Thus, the idea is to take into account these multiples matches in the criterion but weighted by their a posteriori probability. In fact, the proper way to do this is to search for the transformation that maximises the likelihood of the scene knowing only the transformation.

Consider now a random matching matrix A. Each possible matching matrix $A$ has a probability $p(A)=P(\mathbf{A}=A)$ and verifies the previous constraints: $\overline{A_{i j}}=E\left(\mathbf{A}_{i j}\right)=P\left(\mathbf{A}_{i j}=1\right) \in[0,1]$ and $\sum_{j} \overline{A_{i j}}=1$. Finally, since scene points are assumed to be independent: $p(A)=\prod_{i j / A_{i j}=1} p\left(\mathbf{A}_{i j}=1\right)=\prod_{i j}\left(\overline{A_{i j}}\right)^{A_{i j}}$.

Let us start from an a priori probability law given by $p(A)=\prod_{i j}\left(\overline{\pi_{i j}}\right)^{A_{i j}}$ (a relevant choice is the uniform law $\overline{\pi_{i j}}=\frac{1}{N_{\mathcal{M}}}$ ). Using Bayes rule and Eq. 2, we can deduce the joint scene and matching matrix likelihood:

$$
p(\mathcal{S}, A \mid \mathcal{M}, T)=p(\mathcal{S} \mid A, \mathcal{M}, T) \cdot P(A \mid \mathcal{M}, T)=\prod_{i j}\left(\overline{\pi_{i j}} \cdot p\left(s_{i} \mid m_{j}, T\right)\right)^{A_{i j}}
$$

Thus, the likelihood of the scene knowing only the transformation is $p(\mathcal{S} \mid \mathcal{M}, T)=$ $\sum_{\{A\}} p(\mathcal{S}, A \mid \mathcal{M}, T)$. Taking the negative log, we obtain the registration criterion:

$$
C(T)=-\log (p(\mathcal{S} \mid \mathcal{M}, T))=-\sum_{i} \log \left(\sum_{k} \overline{\pi_{i k}} \cdot p\left(s_{i} \mid m_{k}, T\right)\right)
$$




\subsection{From the Maximum Likelihood to the EM Criterion}

This criterion, like the first ICP criterion, has no closed form solution. We construct in this section an auxiliary criterion, depending explicitly on the matching matrix, and we use an alternated optimisation scheme. This construction follows EM principles [3]10] and the auxiliary variables framework of [4].

Designing the Auxiliary Criterion For any matching matrix $A$, we can write the criterion using Bayes rule: $C(T)=-\log p(A, \mathcal{S} \mid \mathcal{M}, T)+\log p(A \mid \mathcal{S}, \mathcal{M}, T)$. Since this is valid for any matching matrix $A$, it is still for the expectation w.r.t. any random matching matrix $\mathbf{A}$ :

$$
C(T)=-E_{\mathbf{A}}(\log p(A, \mathcal{S} \mid \mathcal{M}, T))+E_{\mathbf{A}}(\log p(A \mid \mathcal{S}, \mathcal{M}, T))
$$

To introduce an explicit dependence on the matching matrix, we add to this criterion the Kullback-Leibler distance $E_{\mathbf{A}}(\log p(A)-\log p(A \mid \mathcal{S}, \mathcal{M}, T))$ between the random variable $\mathbf{A}$ and the random variable $\mathbf{A}_{T}$ defined by $P\left(\mathbf{A}_{T}=A\right)=$ $p(A \mid \mathcal{S}, \mathcal{M}, T)$. This distance is null for $\mathbf{A}_{T}$, positive otherwise. Thus, we have:

$$
C(T, \mathbf{A})=-E_{\mathbf{A}}(\log p(A, \mathcal{S} \mid \mathcal{M}, T))+E_{\mathbf{A}}(\log p(A))
$$

with $C(T)=\min _{\mathbf{A}} C(T, \mathbf{A})=C\left(T, \mathbf{A}_{T}\right)$. A natural optimisation scheme for this new criterion is then to alternate an Expectation step to estimate the matching matrix and a Minimization step to solve for the transformation.

E-Step: Estimation of Matches In this step, we want to compute the optimal values $\overline{A_{i j}}$. By definition of our auxiliary criterion, the optimal $\mathbf{A}$ has the pdf:

$$
p\left(A_{T}\right)=p(A \mid \mathcal{S}, \mathcal{M}, T)=\frac{p(A, \mathcal{S} \mid \mathcal{M}, T)}{p(\mathcal{S} \mid \mathcal{M}, T)}=\prod_{i j}\left(\frac{\overline{\pi_{i j}} \cdot p\left(s_{i} \mid m_{j}, T\right)}{\sum_{k} \overline{\pi_{i k}} \cdot p\left(s_{i} \mid m_{k}, T\right)}\right)^{A_{i j}}
$$

Since we have $p\left(A_{T}\right)=\prod_{i j}\left(\overline{\left(A_{T}\right)_{i j}}\right)^{A_{i j}}$, we obtain by identification:

$$
\overline{\left(A_{T}\right)_{i j}}=\frac{\overline{\pi_{i j}} \cdot p\left(s_{i} \mid m_{j}, T\right)}{\sum_{k} \overline{\pi_{i k}} \cdot p\left(s_{i} \mid m_{k}, T\right)}=\frac{\overline{\pi_{i j}} \cdot \exp \left(-\mu^{2}\left(s_{i}, T \star m_{j}\right) / 2\right)}{\sum_{k} \overline{\pi_{i k}} \cdot \exp \left(-\mu^{2}\left(s_{i}, T \star m_{k}\right) / 2\right)}
$$

Contrary to the ICP (see remark at end of 2.1), these values are not invariant w.r.t a global scaling of the noise variance. Hence, the noise variance is an effective parameter of the EM algorithm (see section 3).

M-Step: Registration Now that we have an estimation of the matching matrix, we can optimize the criterion w.r.t. the transformation. In Eq. 4, only the first term depends on the transformation. Therefore we have to minimise $-E_{\mathbf{A}}(\log p(A, \mathcal{S} \mid \mathcal{M}, T))=-\sum_{i j} \overline{A_{i j}} \log \left(\overline{\pi_{i j}} \cdot p\left(s_{i} \mid m_{j}, T\right)\right)$ Discarding constant and normalisation factors, we are left with the minimization of:

$$
\sum_{i j} \overline{A_{i j}} \cdot \mu^{2}\left(s_{i}, T \star m_{j}\right)=\sum_{i j} \overline{A_{i j}} \cdot\left\|s_{i}-T \star m_{j}\right\|^{2} / \sigma_{s}^{2}
$$



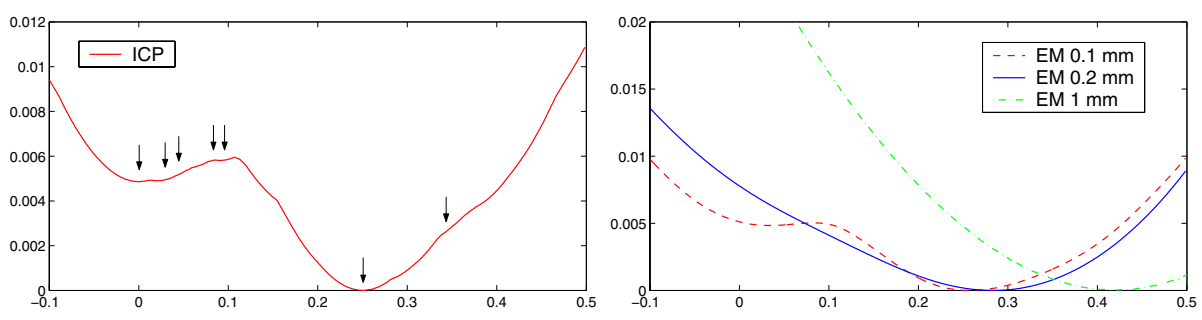

Fig. 1. Criterion vs Z-translation for ICP (on the left) and for EM (on the right). There are two relevant minima for $\operatorname{ICP}(0$, where the algorithm stopped, and 0.25, where criterion is minimum) and irrelevant ones (e.g. 0.1 and 0.3). The EM algorithm has a much smoother shape. For an under-estimated variance $(0.1 \mathrm{~mm})$ the irrelevant minima have disappeared. For the approximative noise variance $(0.2 \mathrm{~mm})$, even the first minima has disappeared. For a larger (overestimated) variance $(1 \mathrm{~mm})$, the criterion is almost quadratic, but the global minimum has been shifted.

Actually, this is the expectation of the standard ICP criterion w.r.t. the random matching matrix, as suggested in the beginning of section 2.2 For the practical optimisation, the only differences with the ICP criterion are the non-binary weights. In the rigid case, this leads to a straightforward adaptation of the SVD or the unit quaternion methods [6].

\section{Practical Use of the EM Algorithm}

In this section, we analyse the role of the variance parameter and its influence on the criterion shape. We investigate different technics to choose this parameter and the impact on the convergence of the algorithm.

Asymptotic Criteria Let us see first how the criterion behaves for asymptotic values of the variance parameter. If the variance goes to zero, it is easy to show [15] that the limit of equation 5 is: $\overline{\left(A_{T}\right)_{i j}}=1$ for the closest point and 0 otherwise. Thus, the ICP algorithm is the limit of the EM algorithm for very small variance parameter. On the contrary, when the variance goes to infinity, the EM algorithm aligns the barycentre and inertia moments [15], generally leading to a shifted result (see Fig. 1). Since under-estimating the variance leads to less accurate results (see section 4) while over-estimating it enlarges the convergence basin, it is logical to start with a large variance, and decrease it to reach the real noise variance.

Setting the Variance Parameter One could think to directly compute the variance after each step of the EM algorithm: considering the variance as an additional parameter and optimising it at fixed transformation and weights leads 
to: $\sigma^{2}=\frac{1}{\operatorname{dim.N_{\mathcal {S}}}} \sum_{i j} \overline{A_{i j}} \cdot\left\|s_{i}-T \star m_{j}\right\|^{2}$. Experiments showed that this estimation is not robust because the decrease is too fast: the algorithm cannot escape the local minima. A slight and regular decrease facilitates the convergence to the global minimum. This can be realised using a deterministic annealing [1] where the variance is multiplied by an annealing coefficient (usually between 0.9 and 0.95) after each iteration until the real noise variance has been reached. This technique allows to avoid local minima to finally reach a very accurate result, but requires a good estimation of the noise variance.

Convergence Since ICP and EM minimise a global criterion alternatively along subsets of the parameters, the criterion always decrease and convergence to a local minimum is ensured. In practice, the algorithm is stopped when the variations of the parameters are small (threshold on the residual transformation). However, the annealing scheme on the variance is not a minimisation step. Thus, the convergence of the algorithm should only be tested after the end of the annealing, when the variance is minimal.

\section{Experiments on Real Teeth Data}

We evaluate in this section the comparative robustness and accuracy of the ICP and EM algorithms on our data. For the robustness, we estimate the size of the convergence basin where an initial transformation leads to the correct global minimum. For the accuracy, we analyse the internal accuracy, due to the presence of local minima in the immediate vicinity of the global minimum, and the global accuracy, due to the noise on the data, the sampling of the surface and possible approximations in our problem modelling.

Algorithm Setup: For all the experiments, we used an annealing coefficient $\alpha=0.9$ with initial variance equal to 16 times the real noise variance $\sigma^{2}$ and a simple outlier rejection using a threshold on the Mahalanobis distance at $\mu_{\max }^{2}=$ 3.dim. The real variance was estimated once on a reliable dataset using the direct variance computation.

Robustness and Internal Accuracy The robustness can be characterised by the size of the attraction basin of the global minimum. However, even if the algorithm converges towards the global minimum, it may be trapped in some small local minima in its immediate vicinity due to errors in the matches estimation. We call internal accuracy this intrinsic variability of the results. The problem is to choose a limit that distinguishes between internal accuracy errors and "uniformly distributed" erroneous convergences outside the attraction basin.

In this experiment, we used a surface segmented from a CT-Scan and a set of 50 points measured on the real jaw. We perturbed 2000 times the results of an "exact" registration by adding uniform translations up to $2 \mathrm{~cm}$ (on these data, we observed that both algorithms were almost insensitive to rotations up to 10 
degrees), used it as initialisation of the ICP and EM algorithms and plot in figure 2 (top) the distance of the estimated to the "exact" transformation. For ICP, (upper left), we observe many local minima, and it is difficult to differentiate a clear structure around 0 from a uniform distribution elsewhere. As rotation and translation are well correlated under $1 \mathrm{~mm}$ and $1 \mathrm{deg}$, we arbitrarily decided that these transformations were representative of the internal accuracy. For the EM algorithm (upper right), the number of local minima has significantly decreased and there is a clear separation between transformations very close to zero and other local minima. Propagating the transformation covariance on test points in the area of interest (the jaw) gives us a measure of the internal accuracy: $0.2 \mathrm{~mm}$ for ICP, and $0.007 \mathrm{~mm}$ for EM.

Now, to determine the size of the attraction basin, we have to look for the smallest initial translation for which the result is classified as bad. We plot in Fig. 2 (bottom left) the percentage of convergence to the global minimum w.r.t. the initial transformation: an initial translation of $2 \mathrm{~mm}$ can lead to bad results with ICP, whereas this limit is shifted to $9 \mathrm{~mm}$ for the EM. Last but not least, we present in Fig. 2 (bottom right) the distributions of the criterion values for both algorithms: there is a clear threshold distinguishing good from bad results with EM, whereas there is no such clustering for ICP. Numerous experiments with various number of points and surface shapes showed that this threshold on EM strongly depends on the noise variance but only slightly on the data shape. Thus, it can be estimated only once for a given application.
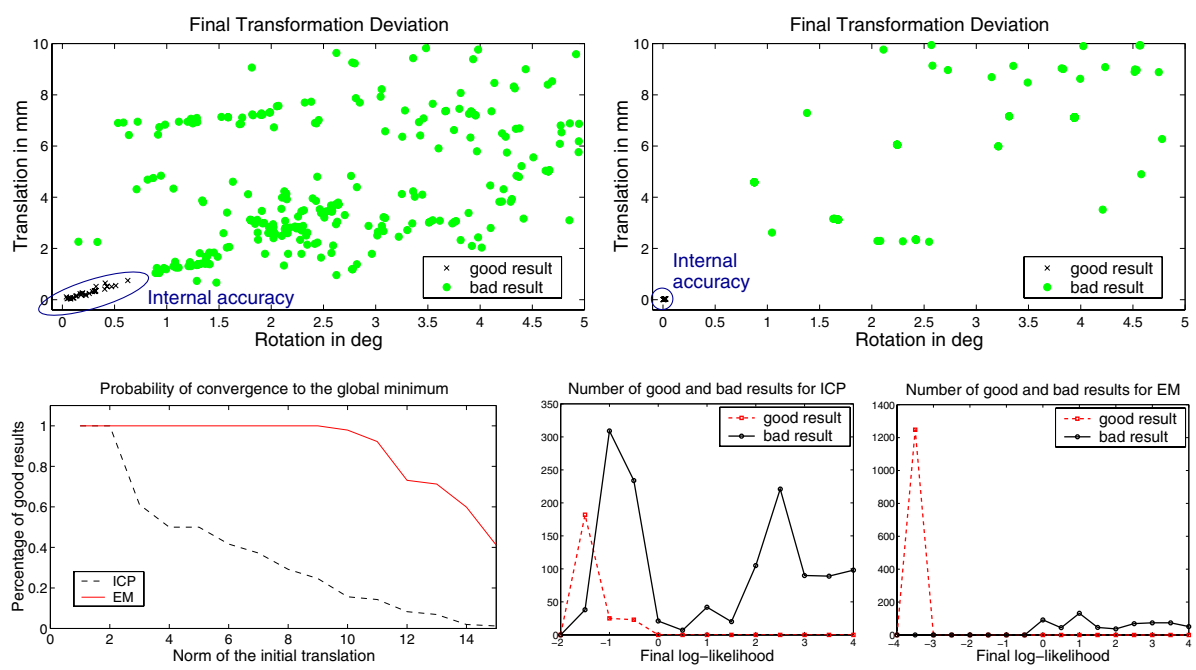

Fig. 2. Top: Final transformations distribution for ICP (on the left) and EM (right). Bottom: probability of convergence to the global minimum with respect to the norm of the initial translation (left), and distribution of the criterion value (right). 

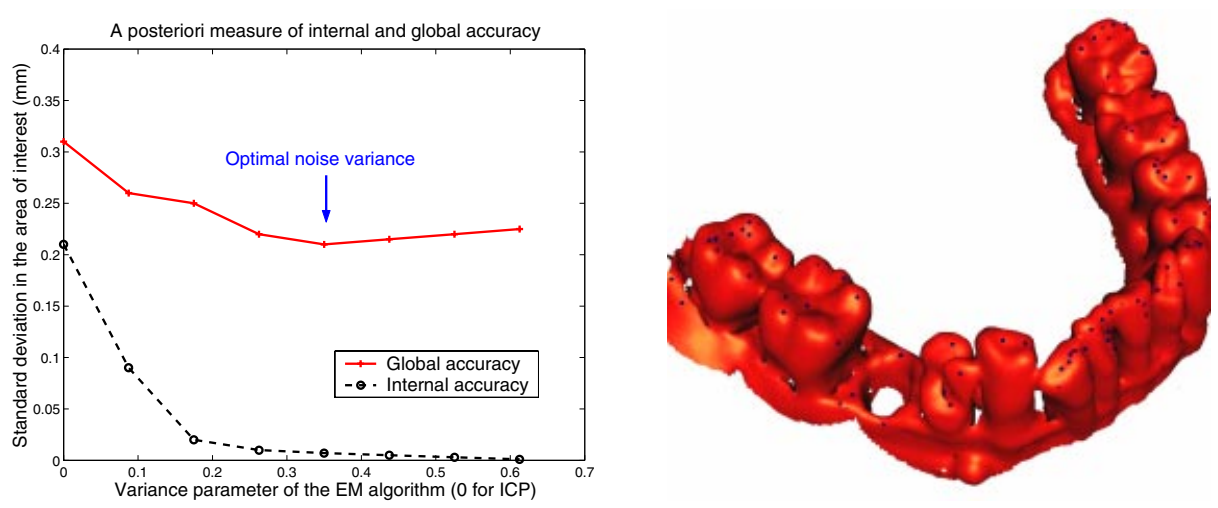

Fig. 3. Left: global and internal accuracy of the EM with respect to the variance. ICP (at $\sigma=0)$ exhibits internal and global accuracies of 0.22 $\mathrm{mm}$ and $0.31 \mathrm{~mm}$, while EM presents at the optimal variance internal and global accuracies of $0.00^{7} \mathrm{~mm}$ and 0.22 $\mathrm{mm}$. Right: $A$ view a the points-surface registration for the optimal variance.

Global Accuracy Evaluation To evaluate the global accuracy (i.e. the difference between the "exact" and estimated transformation) of the algorithms in real conditions, ten sets of 50 scene points, each time randomly placed on the fixed jaw, were acquired and registered onto the same model, segmented from a CT-Scan. Thus, the model variability was not taken into account, but all other sources of errors (segmentation error, scene points measurement error, and especially effect of surface sampling) were realistic. The "exact" registration was determined by the registration of all sets of points together to the model surface: this transformation should have a variance 10 times smaller than individual registrations, but hides a possible bias. Figure 3 presents the standard deviation of test points on the jaw for different values of the variance in the EM algorithm: underestimating this parameter appears to be much more penalising in terms of accuracy than overestimating it.

Registration Time In scope of a peroperative system, the computation time is a key parameter. In both algorithms, this time depends strongly on the number of scene points and iterations, and the distance threshold. Thanks to efficient space-partitioning structures, it only slightly depends on the number of model points. For each iteration (with the same data and distance threshold), EM only adds a $30 \%$ overhead to the ICP time, but it usually needs much more iterations to converge. Typically, it took 50 iterations (including 20 for deterministic annealing) on the above experiment, against 20 for ICP. The final time comparison is four to one in favour of ICP. However, the total computation time of EM is about $30 \mathrm{~s}$ in our case, which is still reasonable for our peroperative system. 


\section{Conclusion}

We present in this article a maximum likelihood approach of the point matching problem and show that looking for both the transformation and the matches leads to the ICP algorithm, while considering the matches as hidden variables gives a new criterion, efficiently solved using an EM method. In this new algorithm, the variance on the data points is an important parameter that allow the EM algorithm to range from a global (alignment of the barycenters and inertia tensors) to a purely local behaviour (ICP). This property is exploited in a deterministic annealing method to avoid local minima while reaching an optimal accuracy.

Experimental results show that ICP has a very small attraction basin (a few millimetres in translation), an important internal error and a global accuracy of $0.31 \mathrm{~mm}$ in the jaw area. The EM algorithm exhibits a much wider attraction basin (around $1 \mathrm{~cm}$ ) with a negligible internal error and a better global accuracy $(0.22 \mathrm{~mm})$. This gain in robustness and accuracy is counterbalanced by a larger computation time (a factor 4), which remains however compatible with our peroperative system.

Future work will include the parallelisation of the algorithm, the study of the surface sampling influence on the accuracy, the use of oriented points (position + normal to the surface) and the online prediction of the registration uncertainty.

Acknowledgements This work was supported by a CIFFRE Ph.D fellowship from AREALL, who also provided all the data used in this study.

\section{References}

1. A. Rangarajan and al. A Robust Point-Matching Algorithm for Autoradiograph Alignment. Medical Image Analysis, 1(4):379-398, 1997.

2. A. Rangarajan, H. Chui and J.S. Duncan. Rigid Point Feature Registration Using Mutual Information. Medical Image Analysis, 3(4):425-440, 1999.

3. C. Couvreur. The EM Algorithm: A Guided Tour. In Proc. of CMP'96, pp.115120, Pragues, Czech Republik, August 1996.

4. L. Cohen. Auxiliary Variables and Two-Step Iterative Algorithms in Computer Vision Problems. J. of Mathematical Imaging and Vision (JMIV), (6):59-83, 1996.

5. D. Etienne, A. Stankoff, X. Pennec, S. Granger, A. Lacan and R. Derycke. A New Approach for Dental Implant Aided Surgery. CARS'2000, pp.927-931, 2000.

6. D.W. Eggert, A. Lorusso and R. Fisher. Estimating 3D rigid body transformations: A comparison of four major algorithms. Mach. Vis. ES App., 9:272-290, 1997.

7. H. Chui and A. Rangarajan. A Feature Registration Framework using Mixture Models. In Proc. MMBIA'2000, pages 190-197, 2000.

8. K. Kanatani. Statistical Optimization for Geometric Computation : Theory and Practice. Elsevier Science (Amsterdam), 1996.

9. P.J. Besl and N.D. McKay. A Method for Registration of 3D Shapes. IEEE Transactions on Pattern Analysis and Machine Intelligence, 14(2):239-256, 1992.

10. R. M. Neal and G. E. Hinton. A View of the EM Algorithm that Justifies Incremental, Sparse, and other Variants. Learning in Graphical Models, 1998. 
11. T. Masuda, K. Sakaue, and N. Yokoya. Registration and Integration of Multiple Range Images for 3D Model Construction. In Proc. ICPR'96, pages 879-883, 1996.

12. W. Wells. Statistical Approaches to Feature-Based Object Recognition. IJCV, 21(1):63-98, 1997.

13. X. Pennec and J.P. Thirion. A Framework for Uncertainty and Validation of 3D Registration Methods based on Points and Frames. IJCV, 25(3):203-229, 1997.

14. Z. Zhang. Iterative Point Matching for Registration of Free-Form Surfaces. IJCV, 13(2):119-152, 1994.

15. Granger et al.. Rigid Point-Surface Registration using Oriented Points and an EM Variant of ICP for Computer Guided Oral Implantology. RR-4169 INRIA, 2001. 